\title{
X.
}

\section{Ueber neuere Beiträge zur Geschichte der Poetik.}

\author{
Von \\ Eugen Wolf in Kiel.
}

Die Regsamkeit unserer litteraturgeschichtlichen Studien hat sich neuerdings auch auf die Geschichte der Poetik ausgedehnt. Nach Karl Borinskis Schrift über „Die Poetik der Renaissance und die Anfänge der litterarischen Kritik in Deutschland“ (1886) wandte sich das Interesse, wie wohl begreiflich, rorwiegend dem 18. Jahrhundert zu. Namentlich liegt, der Zeit nach an jenes Buch anknüpfend, nunmehr eine "Geschichte der poetischen Theorie und Kritik von den Diskursen der Maler bis a uf Lessing von Friedrich Braitmaier vor (2 Theile, Frauenfeld, J. Hubers Verlag, 1888 und 1889). Unmittelbar vorher erschien eine Arbeit über die erste Hälfte dieses Themas allein, unter dem Titel: „Die Poetik Gottscheds und der Schweizer litterarhistorisch untersucht" von Franz Servaes (Quellen und Forschungen zur Sprachund Culturgeschichte der germanischen Völker, Band 60, Strassburg, Karl J. Trübner, 1887). Das Jahr 1889 zeitigte daneben eine Anzahl von Einzelbeiträgen zur Geschichte der Poetik im 18. Jahrhundert; so Oscar Natoliczka: Schäferdichtung und Poetik im 18. Jahrhundert (Vierteljahrschrift für Litteraturgeschichte II, 1ffi.), J. Bystron: Lessings Epigramme und seine Arbeiten zur Theorie des Epigramms (Leipzig, Gustav Fock), Gustav Zimmermann: Versuch einer Schillerschen Aesthetik. (Leipzig, B. G. Teubner), schliesslich Karl Gneisse: Untersuchungen zu Schillers Aufsätzen „Ueber den Grund des Vergnügens an tragischen Gegenständen“, 
"Ueber die tragische Kunst" und "Vom Erhabenen" (Weissenburg i. E., Programm-Abhandlung des Gymnasiums).

Wollen wir prüfen, inwieweit diese Schriften die Wissenschaft fördern, so harrt zunächst die Vorfrage der Erledigung: zur Förderung welcher Wissenschaft sie eigentlich verfasst wurden; denn die Litteraturgeschichte und die Philosophie nehmen gleichermassen Antheil an der Poetik und deren Geschichte. Oder vielmehr wir werden fragen müsssen, ob diese Werke nach beiden gekennzeichneten Seiten hin ihrer Aufgabe gerecht werden; ist doch das Wort Wilhelm Scherers von bedeutsamer Wahrheit: "Zwischen Philologie und Aesthetik ist kein Streit, es sei denn, dass die eine oder die andere oder dass sie beide auf falschen Wegen wandeln. ${ }^{\alpha} \mathrm{Ob}$ gleich also die genannten Schriften aus litteraturgeschichtlichen Kreisen hervorgegangen zu sein scheinen, bleibt zu erwarten, oder doch zu fordern, dass sie auch für die Philosophie Nutzen bringen.

Diese Forderung ist um so nachdrücklicher gerade dann $\mathrm{zu}$ erheben, wenn sich die Verfasser, wie Braitmaier, gegen die bisherige Geschichtschreibung der Aesthetik "wenden. „Ist die dürftige Skizze, die die Geschichten der Philosophie geben“, sagt er II, 143, „wenigstens im ganzen richtig, so ist die betreffende Partie in den Geschichten der Aesthetik, wie überhaupt die Zeit vor Kant, mit geradezu verblüffender Oberflächlichkeit und Unkenntniss der Quellen geschrieben. Man greift beliebig aus der Vorrede oder Einleitung ein paar Sätze heraus, verdreht sie in das Gegentheil ihres Sinnes, zieht Folgerungen daraus, an die der Verfasser nie gedacht - und der Mann ist abgethan. " - Gleich nervös polemisirt der Verfasser indess gegen Danzel, den ersten Litteraihistoriker, welcher das behandelte Gebiet quellenmässig erschlossen hat; in schroffem Widerspruch mit der herrschenden Auffassung sieht er $(I, 4)$ den Unterschied zwischen diesem Forscher und Gervinus darin, „dass Danzel die Geschichte philosophisch construirt, Erscheinungen von unbedeutender Tragweite zu Ideen, die die ganze Entwicklung beherrschen sollen, aufbauscht und so ein Bild des geschichtlichen Verlaufs entwirft, das der Wirklichkeit nicht entspricht. Es fehlt dem Aesthetiker der sichere historische Takt“. - Auch zu der Methode der heutigen Litteraturgeschichte stellt 
sich Braitmajer in Gegensatz durch verweisende Behauptungen wie (cbd.): "Jeder, der die zahlreichen Briefwochsel des vorigen Jahrhunderts kennt, weiss, dass keiner ausser dem Goethe-Schillerschen eine wirkliche Ergänzung der litterarischen Thätigkeit des Schriftstellers bietet."

Dadurch erregte $Z$ weifel an der ruhigen Objectivitït des Buches finden in den ersten, Gottsched gexidmeten Kapiteln dessellen lejcht bestimmte Nahrung: in dem Streben, die ror Danzel über Gottsched herschende Ansicht wiederherzustellen, wird ihm nicht nur Gottsched, sondern auch Danzel zu einer lächerlichen Figur; nach einer directen Bemerkung ( $I, 230)$ scheint Braitmaier sich fast als "Vertreter" der "Sache" der Schweizer zu fühlen. Das sind Temperamentsausbrüche, welche auch den wohlwollendsten Leser zunächst stutzio machen.

Und wohlwollende Leser verdient das Braitmaiersche Buch trotz alledem. Der Ernst und treue Fleiss jahrelanger Hingebung spricht aus ihm ebenso unverkennbar wie die umfangreiche Kenntniss der Poetik in ihrer geschichtlichen Entwicklung und die Fähigkeit selbständiger, verhältnissmässig klarer Darstellung der oft wirren Theorien. In der Zuverlässigkeit dieser positiven Darstellung liegt denn auch der Hauptwerth des Werkes. Aber selbst die Urtheile verdienen ausserhalb der Gottsched gewidmeten Partie meist sicher und treffend genannt zu werden. Namentlich ist der Verfasser von einer absoluten Ueberschätzung der Schweizer weit entfernt, betont vielmehr $(I, 28)$ an Bodmer sehr richtig „die vüchterne Verständigkeit, die Vorliebe für Reflexion und rhetorisches Pathos." Der Geschmack ist für denselben Bodmer noch durchaus Sache des Verstandes (I, 82); charakteristisch ist namentlich, was die Schweizer unter Phantasie verstehen $(I, 66)$ : sie beweise ihre Schöpferkraft in der Allegorie, der äsopischen Fabel und - den Todtengesprächen! Wir sehen in Bodmer und Breitinger eben einen Versuch, mit lahmen Schwingen sich etwas über den Erdboden zu erheben, - das Streben des Verstandes, das instinctiv geahnte Uebersinnliche der Poesie zu begreifen. Andererseits fehlt ihnen nicht allein philosophische Schulung, sondern überhaupt die Fähigkeit zu streng logischem Denken ( $I, 68)$, so dass denn in 
der That ihre Schritten aussergewöhnlich wirr und widerspruchsvoll sind.

Mit Recht stellt Braitmaier ferner Johann Elias Schlegel an dic höchste Stelle der vorbaumgartenschen Zeit, wenn er auch als Quelle von dessen ästhetischen Anschauungen weit mehr als zutroffend die Schriften der Schweizer annimmt; in Wahrheit geht J. E. Schlegel von Gottsched aus; dessen Autorität wird in ihm allerdings mit durch die Schweizer erschüttert, aber über ihn hinaus gelangt er erst mit Hülfe vorgeschrittener französischer Kunstrichter. - Wie leicht Johann Adolf Schlegel gegen diesen seinen Bruder wiegt, kommt ohne Verkennung von dessen Verdienst, das in der Verwerfung der Naturnachahmung als höchsten ästhetischen Grundsatzes besteht, ebenso treffend zur Darstellung wie der Fortschritt, welchen Gellert nach der Seite des Gefühls hin herbeiführt.

Der II. Band hebt mit einer manches Neue bringenden historischen Entwicklung und selbständigen kritischen Würdigung von Baumgartens Grundlegung der eigentlichen Aesthetik an. Es folgt eine schlagende Charakteristik von Sulzers intellectuell-moralisch beschränkter Theorie. Ueberwiegend ist dieser Band Moses Mendelssohn gewidmet; hier gewinnt der Verfasser höchst interessante, zum Theil überraschende Ergebnisse; so namentlich in Gegenüberstellung von Mendelssohn und Herder (II, 134ff.: „So ist Herder ebenso wenig Vertreter des rein modernen Gedankens, als Mendelssohn der blosse Vertreter der alten Schule") sowie im Nachweis von Mendelssohns Einfluss auf Schiller (II, 150f.); mit vollem Rechte erkennt er auch in Mendelssohns Schriften die starke Betonung des philosophischen Gehaltes für die moderne Poesie an und plädirt warm für eine gerechtere Würdigung von Mendelssohns verständnissvollem Eifer auf dem Gebiete des deutschen Geisteslebens. Mit einer anschaulichen und besonders auch gegen Lessing unbefangenen kritischen Charakteristik von Nicolais Abhandlung und Mendelssohn-Lessings Briefwechsel über die Tragödie schliesst das Werk.

Es könnte Verwunderung erregen, in ein und derselben Schrift den Verfasser anfangs als Heisssporn, später als besonnen abwägenden Historiker kennen zu lernen. Indessen hat er schon 
1879 eine Programmabhandlung über ,Die poetische Theorie Gottscheds und der Schweizer" veröffentlicht und hält, wie er betont, an der dort gegebenen Darstellung, mehr als gut war, auch heute noch fest. Er hat aber inzwischen erst geschichtliche Anschaung erworben, scheinbar unbewusst, so dass er verabsäumte, auf Grund derselben die vorgefasste Meinung über scinen Ausgangspunkt Gottsched einer Nachprüfung zu unterziehen.

Diese im grösscrn Theil von Braitmaiers Werk nun vorherrschende geschichtliche Anschauung wird gewiss auch als eigentliches Momont der philosophischen Betrachtung bezeichnet werden dürfon: deren $W$ esen ja eben darin besteht, jeden Geist aus sich sellsst zu begreifen. Es wäre zu wünschen, dass dieses Eindringen in deu geschichtlichen Zusammenhang und Geist der Zeiten sich bis ins Einzelne $\%$ einem ausgeführten Gemälde rundete, dass also die Geschichte der: Poetik eine gleich feste Methode erringt wie die Geschichte der Poesie. Von verstreuten glücklichen Ansïtzen gerade auch in Braitmaiers Werk - abgesehen, gaben die bisherigen Beiträge zur Geschichte der Poetik meist nur positive Charakteristik von Ideen, Darstellung von Theorien: die Litteraturgeschichte ist aber schon dazu vorgeschritten, diese Theorien und Ideen, die doch nichts für sich Bestehendes sind, zu der geschichtlichen Gesammtentwicklung in Beziehung zu setzen und in $\mathrm{Zu}$ sammenhang zu betrachten mit dem, woran sie gebunden sind, mit den Menschen, welche Träger der Ideen sind. Erst dadurch wird vollends klar, wie durch Anlagen und Verhältnisse der Personen die Theorien sich aus- und umbilden; erst dadurch sehen wir die Ideen mit Nothwendigkeit aus dem Geiste der Zeit und der Schaffenden herauswachsen und verlieren jene unphilosophische Anschauung von Zufälligkeit der geistigen Erscheinungen. Es liegt auf der Hand, dass eine solche Darstellung nur auf Grund ausgeführter Einzeluntersuchungen möglich sein wird, so dass an einer heutigen Zusammenfassung grösserer Zeitabschnitte niemand billigerweise den Mangel solcher Bezuguahme rügen darf. Worauf es hier ankam, war, ein Ziel hinzustellen, welches die Geschichte der Poetik zu erstreben hat. Dabei bleibt natürlich der von Brait- 
maier meist wohl beachtote Zusammenhang mit den philosophischen Systemen der Zoit immer in erster Jinic stehen.

So wïrde namentlich die Stellung Gottscheds sich mit wissenschaftlicher Ueberzougungskraft bezeichnen lassen, wenn wir einerscits fragen, inwieweit or einen Fortschritt oder Rückschritt über die deutscho Poesie sowie die deutsche und fremde Poetik in 17. und ersten Viertel des 18. Jahrhunderts herbeiführt, andererseits inwieweit noch die folgende Generation und namentlich Lessing auf seinen Schultern steht. Woher plötzlich dieser ganze Leipziger Dichterkreis? woher Gottscheds vorübergehende Dictatur? Hierin liegt doch ein geschichtliches Problem, und des weiteren ein philosophisches: welche Bedeutung hatte und hat dennoch die Betonung der Naturwahrheit in der Kunst?

Beurtheilen wir indess das Braitmaiersche Werk nach allen Anforderungen, die sich heute an eine orientirende Gesammtbetrachtung eines grösseren Abschnittes aus der Geschichte der Poetik stellen lassen, so ist dasselbe entschieden als nützlich und an vielen Punkten fördernd zu bezeichnen. Ich möchte selbst anerkennen, dass der kalte Wasserstrahl, mit welchem der Verfasser Gottsched und dessen verdienten Retter Danzel überschüttet, für den künftigen Biographen des litterarischen Dictators sein Heilsames hat: es ist wenigstens zutreffend, dass Danzel, unter Beiseitelassung der Werke, fast ausschliesslich auf den Briefen an Gottsched fusst; und ich halte nach dem nüchternen, wenn auch zu schroffen und unhistorischen Urtheil Braitmaiers die Gefahr einer Ueberschätzung Gottscheds nach der rein geistigen Seite wesentlich verringert. Als ein Verdienst darf es der Verfasser namentlich in Anspruch nehmen, durch Heranziehung der frühen Zeitschriften Gottscheds und der Schweizer die Entwicklung ihrer Theorien, allerdings nur so lange sie führende Geister sind, veranschaulicht zu haben.

Dennoch verliert die Schrift von Servaes über denselben Gegenstand nicht ganz ihren Werth, soweit ihr ein solcher überhaupt innewohnte. Man darf denselben in der ansprechenden und anschaulichen allgemeinen Kennzeichnung des Gottschedschen und schweizerischen Systems sehen; von einer geschichtlichen Entwick- 
lung ist Servaes indess so weit entfernt, dass er z. B. nicht einmal dic 1. Auflage von Gottscheds Critischer Dichtkunst eingesehen lat. Mancherlei hübsche Bemerkungen laufen unter; namentlich sind die von Danzel schon angedeuteten. Bezichungen za den Philosophien von Leibniz, Wolf und besonders auch Gottsched sellst consequent verfolgt; er verweist mit Recht auf den merkwürdigen Vortrag Gottscheds „Ob man in theatralischen Gedichten allezeit die Tugend als belohnt und das Laster bestrafet vorstellen müsse“, worin Gottsched das frïher anerkannte Moralitätsprincip zu Gunsten unumschränkter Durchführung seines Nachahmungsprincipes aufgiebt; er bringt einmal verständnissvoll die Theorien mit der psychologischen Anlage der Menschen zusammen, - aber im ganzen ist die Schrift doch nicht tiefer eindringend, manche Aeusserungen, die an sich in richtiger Linie liegen, werden in ihrer verallgemeinernden Form falsch oder zum mindesten schematisch, und namentlich das Kapitel „Litteraturfehde“ ist gar zu oberflächlich abgethan.

Eine sehr interessante Ergänzung erfährt Braitmaiers Zusammenfassung durch Netoliczkas fleissige und gründliche Behandlung der Thcorie des Idylls. Wirksam ist die zusammenhängende Betrachtung von Theorie und Production: sie beeinflussen sich in der That fast immer. Als Ausgangspunkt der Untersuchung dient Johann Adolf Śchlegels Aufsatz „Der eigentliche Gegenstand der Schäferpoesie"; nicht nur das Verhältniss zu andern deutschen Stimmen, sondern auch zu Fontenelle und dem Guardian wird klargelegt, namentlich aber Gottscheds Stellung zn dieser Frage charakterisirt. In Uebereinstimmung mit Braitmaier sieht der Verfasser als ersten bedeutsamen Fortschritt die Kritik Mendelssohns über J. A. Schlegel in den Litteraturbriefen an. Treffend kommt schliesslich zur Geltung, wie erst Herders litteraturvergleichender Blick den Abstand zwischen Gessner und Theokrit markirte. Daran schliessen sich (allerdings nur kurze) Hinweise auf Schillers entscheidende Untersuchungen über das Idyll im Aufsatz über naive und sentimentalische Dichtung sowie auf Aeusserungen der Söhne J. A. Schlegels, der beiden Romantiker.

$\mathrm{Zu}$ Herder führt uns auch Bystrons Schrift, welche in einer 
Gegenüberstcllung von Lessings und Herders Epigrammentheorie gipfelt: Lossings Muster Martial, Herders classischeres die grie-. chische Anthologie; Lessing kennt nur eine wahre und höchste Gattung des Epigramms, Herder unterscheidet sieben, und bildet sich“, wie er erklärt, „, noch nicht ein, jede epigrammatische Schönheit mit diesen Abtheilungen gefesselt zu haben". - Es ist erfreulich, durch diese Abhandlungen den Blick über Lessing hinaus auf Herder gerichtet zu sehen, welcher durch die kanonische Bedeutung, zu welcher Lessing in ausserphilosophischen Kreisen noch vielfach erhoben wird, allzusehr in den Schatten trat.

Schillers ästhetische Schriften dagegen bilden seit lange ein Lieblingsthema kunsttheoretischer Studien, freilich mehr im allgemeinen Interesse der Philosophie und Aesthetik als im besondern der Poetik. Es fehlt noch an einer Darstellung, welche das Verhältniss von Schillers Aufsätzen zu der specifisch dichterischen Theorie und Production grundsätzlich verfolgte. Dann würde seịn Herauswachsen aus der Sturm- und Drangperiode und deren geistigen Vätern, sein Abstand von Lessing, sein näheres Verhältniss zu der französischen Tragödie, vor allem der Zusammenhang seiner eigenen Dichtungen mit seinen Anschauungen über das Wesen der Kunst, ja ihre gegenseitige Beeinflussung offenbarer werden. Die Eingangs citirte Schrift von Gustav Zimmermann giebt wenigstens Hinweise auf Young, 'Wood u. a., aber ohne consequente Verfolgung von Schillers Berührung mit ihnen. Ueberhaupt bietet die mit rühmenswerther Begeisterung für das Schillersche Ideenleben verfasste Schrift mehr einen rhetorischen Ueberblick über Schillers theoretisches Gebäude als eine Fortführung der bisherigen Forschungen.

Zimmermann bestreitet mit Tomaschek die Darlegung Kuno Fischers, dass Schiller seit der Verbindung mit Goethe.den ästhetischen Gesichtspunkt über den moralischen gestellt habe. Gneisse dagegen, dessen Abhandlung den für unsere Zwecke vielverheissenden Nebentitel "ein Beitrag zur Kenntniss von Schillers Theorie der Tragödie" führt, sucht nachzuweisen, dass unser Aesthetiker dem Vergnügen am Tragischen keine moralische Begründung gegeben. Sie haben, scheint mir, in gewissem Sinne beide Recht. 
Schiller ordnet zwar immer noch das Moralische dem Aesthetischen cin, aber dieses Moralische liegt zunächst im tragischen Helden, erst mittelbar im Zuschauer. Dessen unmittelbare Empfindung ist, wic Schiller unter Abweichung von Aristoteles mit feinem psychologischen Verständniss feststellt, eine sympathetische, welche durch die blosse Vorstellung des Leidens von Unlust befreit ist. Aber hat Schiller die positive Lust an tragischen Gegenständen gleich befriedigend erklïrt? - Jedenfalls zeigt sich Gneisse, nobeuher manchen aus Missverständniss hervorgegangenen Tadel zurückweisend, als unbeirrter und geschickter Interpret von Schillers Anschauungen.

Alles in allem ist sonach die Thätigkeit zur Geschichte der Poetik eine mannigfach anregende und fördernde; den meisten der besprochenen Autoren wird man gern auf gleichem Gebiete wiederbegegnen. Ueberdies ist hiermit ein Boden mehr gewonnen, auf welchem Litteraturgeschichte und Philosophie Hand in Hand gehen; durch gemeinsame Arbeit kann aber dicse vor Verflüchtigung, jene vor Verknöcherung bewahrt bleiben. 